\title{
Comparação de diferentes indicadores da comunidade biológica no reservatório de Volta Grande, Rio Grande-MG/SP
}

Os diversos componentes da biota reagem com maior ou menor sensibilidade aos diferentes tipos de impacto antropogênico, de forma que demonstram de maneira mais realista, a verdadeir situação dos corpos d' água. Contudo, basear-se em apenas uma comunidade muitas vezes não é suficiente para caracterizar o ambiente como um todo, pois o resultado será de acordo com as particularidades da mesma. $\mathrm{O}$ objetivo deste trabalho foi comparar indicadores da comunidade biológica no reservatório de Volta Grande, Rio Grande MG/SP e suas implicações n avaliação da qualidade da água. No período de abril de 2013 a maio de 2015, nove campanhas de amostragem foram realizadas, em três transectos com três pontos cada. Em cada ponto coletado, foram amostrados parâmetros físico-químicos além das comunidades fito, zooplanctônica e bentônica. Foram calculados os principais índices de qualidade de água e bióticos, sendo os mesmos correlacionados entre si. Considerando Índice de Estado Trófico, a maioria dos valores calculados ocorre abaixo de 47, limite superior para ultraoligotrofia, com exceção do ponto 4A que apresentou valores de oligotrofia. Considerando a comunidade fitoplanctônica, os fitoflagelados da classe Cryptophyceae foram os que mais contribuíram para a densidade, seguidos pelas classes Bacillariophyceae e Chlorophyceae. De acordo com o Coeficiente Múltiplo, as espécies associadas a ambientes enriquecidos, indicaram o ambiente como eutrofizado. Para a comunidade zooplanctônica, razão Calanoida/Cyclopoida classificou a maioria das amostras do transecto 5 como oligotróficas. Para os transectos 3 e 4 , os valores observados geralmente estiveram abaixo de 1, classificando estes trechos como meso-eutróficos. O índice BMWP apresentou valores entre 1 e 29 para os organismos bentônicos do reservatório de Volta Grande, classificando os pontos como ruim ou muito ruim. Dentre todas as correlações entre os índices de qualidade de água e bióticos, apenas aquela entre a razão Calanoida/Cyclopoida e o BMWP/Minas foi significativa, ainda que o coeficiente seja baixo $(-0,3476)$. A correlação entre índices de qualidade de água deve ser examinada com cautela, pois dependendo da faixa de variação de suas classificacães, a pontuacão baixa pode indicar boa qualidade de água (como IET, Coeficiente Múltiplo e razão Calanoida/Cyclopoida) ou má qualidade (no caso do BMWP). indicadores da comunidade biológica no reservatório de Volta Grande, permitiu concluir que a aplicação desses índices em conjunto traz um resultado com maior grau de assertividade, uma vez que a boa qualidade físico-química da água não reflete a degradação das comunidades biológicas.

\section{Comparison of different indicators of biological community in Volta Grande reservoir, Grande River-MG/SP}

\begin{abstract}
Various components of biota react with more or less sensitivity to different types of anthropogenic impacts, showing, more realistically, the true state of water bodies. However, considering only one biotic community is often not enough to characterize the environment as a whole, because the result will be according to the particularities of the environment. The objective of this study was to compare biological community indicators in Volta Grande reservoir, Grande River, MG/SP, and its implications for water quality assessment. In the period from April 2013 to May 2015, nine sampling periods were collected in three transects of three stations each. At each station collected, physical-chemical parameters were sampled in addition to the phyto, zooplankton and benthic communities. The main water quality and biotics indexes were determined, being the correlated among them. Regarding the Trophic State Index, most of the calculated values occur below 47 , upper limit for ultraoligotrophy, except thestation $4 \mathrm{~A}$ that presented values of oligotrophy. Considering the phytoplankton community, the phytoflagellates
of Cryptophyceae were the ones that most contributed to density, followed by Bacillariophyceae and Chlorophyceae. According to the Multiple Coefficient, these species associated with enriched environments indicated the eutrophy. For the zooplankton community, the Calanoida/Cyclopoida Ratio classified most of the transect 5 samples as oligotrophic. For transects 3 and 4, most of calculated valueswere below 1, classifying these stretches as meso-eutrophic. The BMWP index showed values between 1 and 29 for benthic organisms of Volta Grande reservoir, classifying the stations as bad or very bad. Only the correlation between Calanoida/Cyclopoida Ratio and BMWP/Minas was significant, however the coefficient was very low (-0.3476). Correlations between water quality indexes should be examined with caution, because depending on the range of variation of their classifications, low scores may indicate good water quality (such as TSI, Multiple Coefficient and Calanoida/Cyclopoida Ratio) or poor quality (as BMWP). Although the physical-chemical parameters classify the Volta Grande reservoir with good quality, the biog indexes together results in a higher degree of assertiveness, since the physical-chemical quality of the water does not reflect the degradation of biological communities.
\end{abstract}

Keywords: Bioindicators; Water Quality Index; Plankton; Zoobenthos; Reservoirs.

Topic: Área temática.

Reviewed anonymously in the process of blind peer.

Gizele Cristina Teixeira de Souza

Universidade Federal de Ouro Preto, Brasil

http://lattes.cnpq.br/6399413215475489

gizelechris@yahoo.com.br

\section{Sofia Luiza Brito}

Universidade Federal de Minas Gerais, Brasil

http://lattes.cnpq.br/7971536190458140

sofialuizabrito@gmail.com

Vera Lúcia de Miranda Guarda

Universidade Federal de Ouro Preto, Brasil

http://lattes.cnpq.br/1324927877730882

vera.guarda@gmail.com

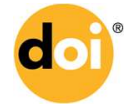

DOI: $10.6008 / S P C 2179-6858.2017 .003 .0006$
Received: 05/02/2017

Approved: 14/04/2017
Referencing this:

SOUZA, G. C. T.; CARVALHO, M. D.; BRITO, S. L.; GUARDA, V. L. M.. Comparação de diferentes indicadores da comunidade biológica no reservatório de Volta Grande, Rio Grande-MG/SP. Revista IberoAmericana de Ciências Ambientais, v.8, n.3, p.52-63, 2017. DOI: http://doi.org/10.6008/SPC2179-6858.2017.003.0006 


\section{INTRODUÇÃO}

Os índices de qualidade de água são amplamente utilizados na caracterização de ambientes aquáticos por sintetizar toda a informação gerada pelo grande número de parâmetros utilizados em monitoramento destes ecossistemas, auxiliando na tomada de decisão e gestão dos recursos hídricos. Por outro lado, os diversos componentes da biota reagem com maior ou menor sensibilidade aos diferentes tipos de impacto antropogênico, de forma que demonstram de maneira mais completa e realista, a verdadeira situação dos corpos d'água.

Nesse sentido, alguns pesquisadores consideram que apenas os parâmetros físicos e químicos não são suficientes para verificar a qualidade ambiental de maneira eficaz, uma vez que frequentemente representam condições momentâneas do ecossistema (KARR et al., 1986; METCALFE, 1989; CALLISTO et al., 2005). Assim, as análises biológicas complementam as físico-químicas e, juntas, formam a base para uma avaliação mais precisa da qualidade das águas correntes (ROUND, 1991; ROUND, 1993).

Por essa razão, recomenda-se o uso de índices bióticos uma vez que organismos aquáticos passam toda ou parte de sua vida neste ambiente, estando sujeitos às condições e aos impactos que os ecossistemas são submetidos. Desta forma, um crescente número de trabalhos tem apontado espécies bioindicadoras de qualidade de água entre os macroinvertebrados aquáticos (HILSENHOFF, 1988), zooplâncton (GANNON et al., 1978; SLADECEK, 1983), fitoplâncton (KELLY et al., 1995; REYNOLDS, 2002) e em outros grupos.

Os organismos planctônicos são importantes indicadores de impactos no curto prazo. Contudo, as mesmas características do fitoplâncton como bioindicador de alterações ambientais (ciclo de vida curto e rápida reprodução), também podem gerar conclusões incorretas, quando se observa apenas variações pontuais, ao curto prazo. Desta forma, Esteves (2011) ressalta a importância de que levantamentos envolvendo a comunidade fitoplanctônica, para determinar estados tróficos, devem ser realizados com pesquisas ao longo prazo.

Os organismos do zooplâncton, geralmente, possuem uma reprodução rápida e a maioria deles possui ciclo de vida curto. Essas características permitem que esse grupo responda rapidamente às alterações ocorridas no ambiente no qual esteja inserido, sendo utilizados inclusive em ensaios ecotoxicológicos. Eles são muitos sensíveis aos impactos ambientais, os quais alteram sua abundância e composição de espécies (COELHO-BOTELHO, 2003).

São diversas as razões para a utilização de macroinvertebrados bentônicos em programas de biomonitoramento da qualidade de água: possuem maior tamanho corporal quando comparados aos organismos do plâncton, assim podem ser vistos a olho nu; possuem hábito sedentário, sendo, portanto, representativos de características locais. Muitos deles vivem nos sedimentos, onde há tendência de bioacumulação de toxinas, e assim podem responder aos impactos de longa duração. Suas comunidades possuem grande diversidade biológica, o que os permitem responder com maior variabilidade diante de diversos tipos de impactos ambientais. Participam da cadeia alimentar, sendo um elo entre os produtores primários e os peixes, desempenhando um papel importante no processamento de matéria orgânica e ciclagem de nutrientes (ROSENBERG et al., 1993; WARD et al., 1995; REECE et al., 1999; Callisto et al., 2001). 
Os principais programas de monitoramento dos ambientes aquáticos em diversos países demandam o emprego dos bioindicadores paralelamente à análise físico-química da água (COPAM, 2008). Contudo, basear-se em apenas uma comunidade, muitas vezes, não é suficiente para caracterizar o ambiente como um todo, pois o resultado será de acordo com as particularidades da mesma. Por esta razão, é importante levar em consideração mais de uma comunidade ou compartimento.

Os três grupos (fitoplâncton, zooplâncton e macroinvertebrados bentônicos) possuem espécies indicadoras de qualidade ecológica, o que potencializa a assertividade dos resultados obtidos no biomonitoramento ambiental. Neste contexto, o objetivo deste trabalho foi comparar indicadores da comunidade biológica no reservatório de Volta Grande, Rio Grande/MG-SP e suas implicações na avaliação da qualidade da água.

\section{METODOLOGIA}

\section{Universo do Estudo: Reservatório de Volta Grande MG/SP}

O Rio Grande, localizado entre os estados de Minas Gerais e São Paulo, tem um sistema de 12 reservatórios em cascata, a fim de otimizar a utilização do potencial hidrelétrico da bacia que representam 7,5 \% da capacidade de geração de energia no Brasil. Os principais impactos do reservatório de Volta Grande são: remoção da mata ciliar, poluição difusa pela agricultura extensiva no entorno, especialmente cana de açúcar, pecuária e cultivos irrigados por pivôs. A contaminação por fontes pontuais ocorre principalmente pela proximidade de áreas urbanas apresentando lançamento de lixo e de esgotos clandestinos não tratados; e industrial com possível contaminação por drenagem superficial do Polo Industrial de Uberaba.

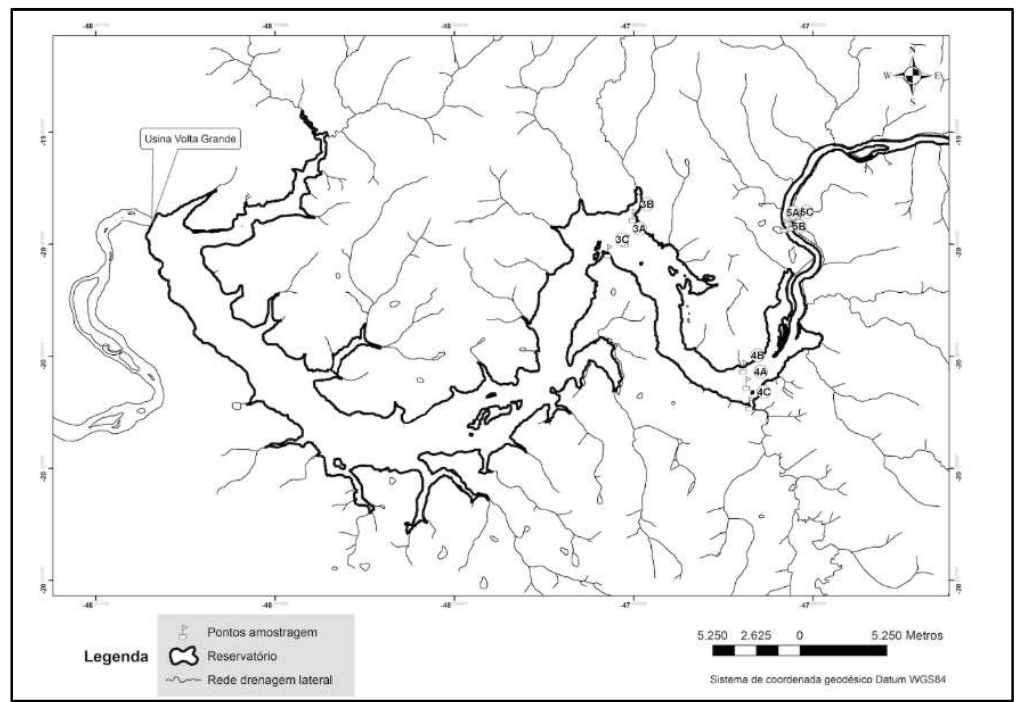

Figura 1: Reservatório de Volta Grande, bacias de contribuição direta e pontos da rede de amostragem. Fonte: IGTEC (2015).

O reservatório de Volta Grande, ilustrado na figura 1 sob as coordenadas $20^{\circ} 02^{\prime} 05^{~ " S ~} 48^{\circ} 13^{\prime} 22^{\prime \prime} \mathrm{W}$, é o nono da cascata do Rio Grande. O lago formado em 1974 ocupa uma área de $205 \mathrm{Km}^{2}$ e uma profundidade máxima de $2 \mathrm{~m}$. Sendo do tipo fio d'água, possui tempo de residência estimado em 2,1 dias. A rede de amostragem na área de influência da UHE Volta Grande foi composta por 3 transectos, cada um abrangendo 
três estações de coleta: região limnética ou calha central (A); margem direita (B); e margem esquerda (C). $O$ transecto 5, localizado no trecho lótico do reservatório, está sob influência do Complexo Industrial de Uberaba/MG e do Ribeirão Conquistinha, cuja bacia drena parte do município de Delta/MG. Os transectos 3 e 4 encontram-se na região de transição do reservatório. No entorno do transecto 3 , observa-se a concentração de pivôs centrais, além da desembocadura do córrego Ponte Alta, sob influência da área urbana de Uberaba/MG. No transecto 4, deságua o Rio do Carmo, principal afluente do reservatório, e encontra-se um vasto banco de Egeria najas, que se encontra no ponto 4A.

No trecho superior (entre os transectos 4 e 5), também foi identificada a dragagem de areia, que revolve o sedimento, causando ressuspensão de sólidos e nutrientes. A introdução de espécies exóticas de peixes (Oreochromis niloticus, Ictalurus punctatus, Clarias gariepinus) (PAIVA et al., 2002) e moluscos (Limnoperna fortunei, Corbicula sp, Melanoides tuberculatus, Physa sp e Biomphalaria straminea) (ELETROBRÁS FURNAS, 2012) tem comprometido a biodiversidade destes grupos de organismos no reservatório.

No período de abril de 2013 a maio de 2015, nove campanhas de amostragem foram realizadas: abril/2013, julho/2013, outubro/2013, janeiro/2014, maio/2014, julho/2014, novembro/2014, janeiro/2015 e maio/2015. As amostras coletadas foram avaliadas físico-quimicamente e bioticamente, no que tange aos seus parâmetros.

A sonda multiparâmetros Horiba U50 foi utilizada para as determinações de pH, condutividade elétrica, oxigênio dissolvido, turbidez, temperatura da água, sólidos totais dissolvidos e potencial redox, sendo as medidas efetuadas in situ na subsuperfície. As amostras de água para a determinação de nitrogênio, fósforo e clorofila a foram coletadas em frascos de $5 \mathrm{~L}$ os quais foram identificados e acondicionados em caixas térmicas até serem encaminhados ao laboratório do UNESCO - HidroEX em Frutal/MG para posterior processamento e análise. Os nutrientes foram analisados segundo as recomendações especificadas no Standard Methods of Water and Wastewater. O Índice de Estado Trófico (IET) médio, considerando concentrações de clorofila $a$, fósforo total e profundidades do disco de Secchi, foi calculado segundo as recomendações de Lamparelli (2004).

Para amostragem da comunidade fitoplanctônica foi coletado $1 \mathrm{~L}$ de água na camada sub-superficial sendo fixado com lugol acético. A comunidade zooplanctônica foi amostrada por meio de arrastos verticais na coluna d'água na região limnética do reservatório e na calha central da região de transição. Nas margens, as amostras foram obtidas na subsuperfície por intermédio da filtragem de $100 \mathrm{~L}$ de água. As amostras foram fixadas em formol 10\% e armazenadas em frascos plásticos. As análises do fitoplâncton e zooplâncton foram realizadas em microscópio ótico em câmara de Sedgewick-Rafter. A comunidade fitoplanctônica foi avaliada através do Coeficiente Múltiplo ou Composto, índice que permite avaliar o estado trófico desta comunidade (NYGAARD, 1949). Este coeficiente considera o número de espécies de Cyanophyceae, Chloroccocales, Centrales e Euglenales, dividido pelo número de espécies de Desmidiaceae, onde valores $<1=$ lago oligotrófico, e se $\mathrm{Q}>1$ = lago eutrófico. A razão Calanoida/Cyclopoida é um índice da comunidade zooplanctônica que tem sido utilizado para indicar alterações do nível de estado trófico de lagos e 
reservatórios (TUNDISI, 1988), onde valores acima de 1 indicam ambientes oligotróficos e abaixo de 1 , mesoeutróficos.

Os macroinvertebrados bentônicos foram coletados na calha central com draga do tipo Eckman com $225 \mathrm{~cm}^{2}$ de área. A amostragem seguiu a padronização de duas dragas por cada estação de coleta $\left(450 \mathrm{~cm}^{2}\right)$. Nas regiões marginais, optou-se pelo uso de uma rede do tipo kicknet com malha de $250 \mu \mathrm{m}$. As amostras foram acondicionadas em sacos plásticos e fixadas com formol bruto. No laboratório, foram lavadas em água corrente sendo despejadas nas peneiras sobrepostas, com $1000 \mu \mathrm{m}$ na parte inferior e $2000 \mu \mathrm{m}$ na parte superior. O conteúdo retido nas peneiras foi transferido para bandejas separadas e observado com lupa e microscópio estereoscópico, averiguando a existência de organismos e a identificação dos mesmos.

Para os macroinvertebrados bentônicos foi considerado o BMWP - Biological Monitoring Working Party, utilizando-se a interpretação deste índice segundo o CETEC (2007) e dos grupos funcionais (Marques et al., 1999; Goulart et al., 2003). Para avaliar se os índices se complementam ou algum se destaca na identificação de impactos ou na caracterização da qualidade de água, foi empregada uma correlação de Spearman (uma vez que maioria dos índices aplicados não apresentou distribuição normal) considerando IET, Coeficiente Múltiplo, Razão Calanoida/Cyclopoida e BMWP.

\section{RESULTADOS}

O reservatório de Volta Grande caracteriza-se por águas bem oxigenadas (média 7,9mg/L); enquanto o $\mathrm{pH}$ apresentou valores abaixo de 7 no transecto 5 e valores neutros nos transectos 3 e 4 . A temperatura média da água variou desde $23,2^{\circ} \mathrm{C}$ na estação seca até $27,2^{\circ} \mathrm{C}$ na estação chuvosa. A transparência apresentou um aumento nas profundidades do disco de Secchi, desde a região lótica (média de 2,01m) até o transecto 3 (média de 2,54m).

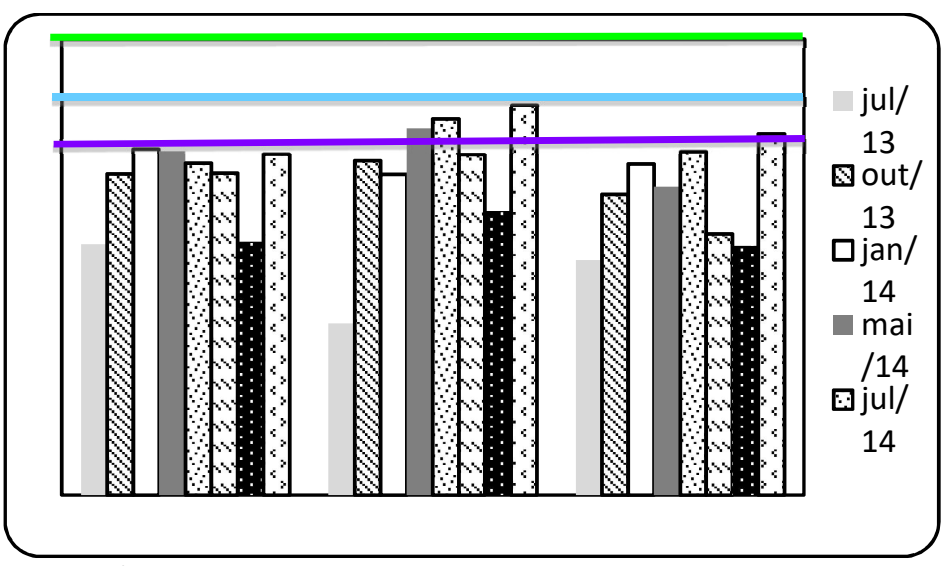

Figura 2: Valores médios do IET - Índice de Estado Trófico, na região limnética em todos os transectos do reservatório de Volta Grande entre julho/13 e maio/15.

Os valores médios de condutividade elétrica $(47 \mu \mathrm{S} / \mathrm{cm})$, turbidez (1,9 NTU), sólidos totais dissolvidos (29 mg/L) corroboram este aumento da transparência ao longo dos transectos. Os valores médios de nitrogênio total $(604,6 \mathrm{mg} / \mathrm{L})$, fósforo total $(13,2 \mu \mathrm{g} / \mathrm{L})$ e clorofila a $(0,67 \mu \mathrm{g} / \mathrm{L})$ foram baixos, indicando uma boa qualidade de água. Quanto ao Índice de Estado Trófico (IET), a maioria dos valores calculados ocorre abaixo de 47 , limite superior para ultraoligotrofia, conforme predisposição na figura 2. A exceção foi o ponto 
$4 A$, que apresentou valores de oligotrofia $(47<$ IET $\leq 52)$ nos meses de maio e julho de 2014 e maio de 2015; além do ponto $5 \mathrm{~A}$ também em maio/2015.

Considerando a comunidade fitoplanctônica, os fitoflagelados da classe Cryptophyceae foram os que mais contribuíram para a densidade (de 37,9 até 100,0 \% da abundância da comunidade), seguidos pelas classes Bacillariophyceae e Chlorophyceae. Com relação ao coeficiente múltiplo, esboçaso na figura 3, não foi possível seu cálculo para todos os pontos uma vez que não foram observadas desmidiáceas em diversas amostras, especialmente no transecto 5. Por outro lado, estavam presentes neste ambiente, espécies associadas a ambientes enriquecidos, sendo que os valores variaram de 1,0 a 17,0 indicando ambiente eutrofizado.

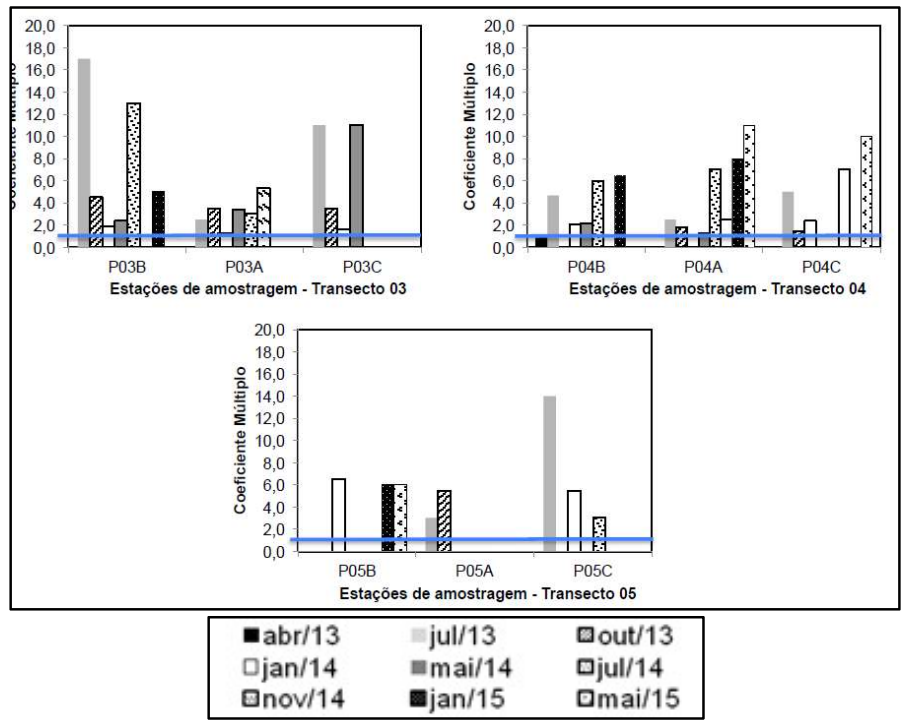

Figura 3: Variação espaço-temporal do Coeficiente Múltiplo de na rede amostral do reservatório de Volta Grande entre julho/13 e maio/15.

Quanto à comunidade zooplanctônica, copépodes das ordens Calanoida e Cyclopoida foram mais abundantes, seguidos de Rotifera e Protozoa, nos transectos 3 e 4, respectivamente. No transecto 5 predominaram as formas naupliares e amebas testáceas, evidenciando sua melhor adaptação ao trecho fluvial do sistema. Maiores densidades foram observadas nos transectos $3\left(38,84 \mathrm{org} . \mathrm{L}^{-1}\right.$, ponto $\left.3 \mathrm{C}\right)$ e 4 ( 22,86 org. $\mathrm{L}^{-1}$, ponto $4 \mathrm{~B}$ ) em mai/14. Por se tratar de um trecho lótico com predomínio de náuplios, não foi possível calcular a razão Calanoida/Cyclopoida para todas as amostras no transecto 5, quando obtidos,verificaram-se valores acima de 2, classificando este trecho como oligotrófico, delineado na figura 4. Para os transectos 3 e 4 , os valores observados geralmente estiveram abaixo de 1 , classificando estes trechos como mesoeutróficos.
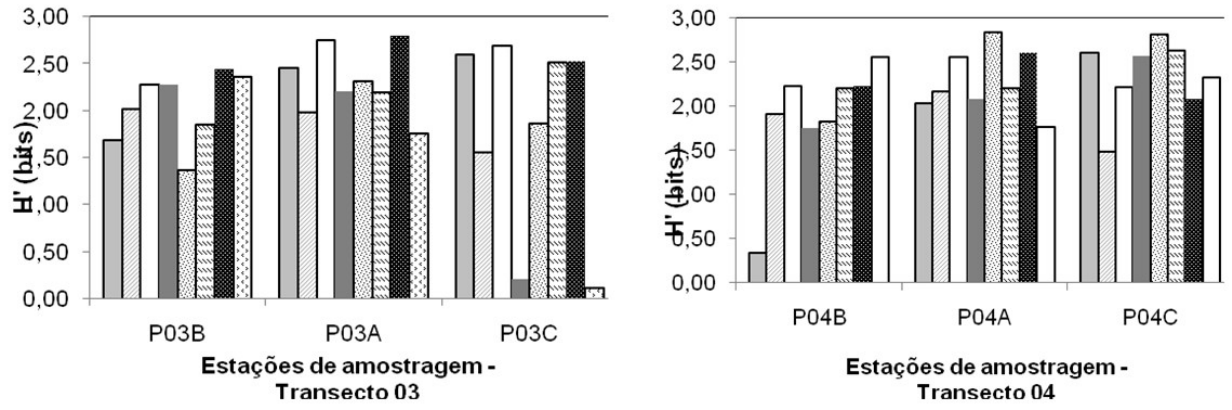


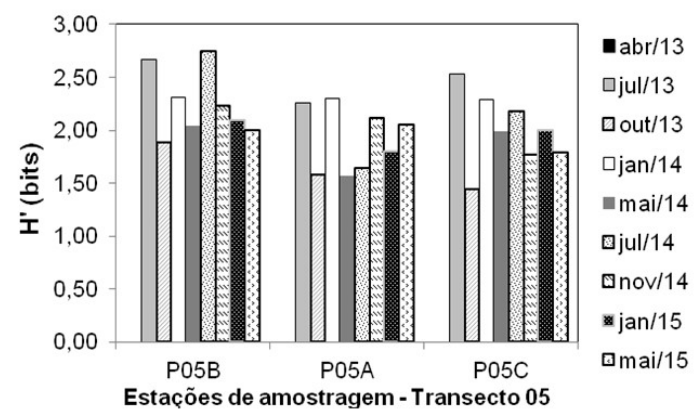

Figura 4: Variação espaço-temporal da Razão Calanoida/Cyclopoida na rede amostral do reservatório de Volta Grande entre julho/13 e maio/15.

Para os macroinvertebrados bentônicos, maior abundância foi observada nas margens, sendo o ponto $4 \mathrm{~B}$ aquele que apresentou os valores mais altos, chegando a 617 e 408 organismos entre janeiro e maio de 2014, respectivamente, seguido do ponto 3C, com 359 organismos em julho/2014. Dentre os grupos funcionais de alimentação existentes, foram identificadas cinco categorias na comunidade de macroinvertebrados do reservatório de Volta Grande: filtradores (Ostracoda, Corbicula sp., Limnoperma fortunei e Oligochaeta), raspadores (Melanoides tuberculatus,Biomphalaria straminea, Physa cf. acuta e Aylacostoma sp.), predadores (Odonata, Ceratopogonidade, parte dos Chironomidae) e coletores (Macrobrachium sp., parte dos Chironomidae) e fragmentadores (Ephemeroptera).

O índice BMWP apresentou valores entre 1 e 29 para as comunidades do reservatório de Volta Grande, como se pode notar na figura 5. A maioria dos pontos foi classificada como muito ruim (69 das ocorrências, $96 \%$ das amostras) ou ruim (3 ocorrências, 4\% das amostras), e nem um único registro de qualidade de água regular. O padrão espacial encontrado com a análise dos resultados do índice é semelhante àquele já registrado para abundância, qual seja, os maiores valores foram registrados na zona litorânea enquanto na calha central ou região limnética, o índice apresenta um resultado pior. A exceção a esse padrão é novamente o transecto 4 cujo ponto 4C (desembocadura do Rio do Carmo), região litorânea, mostrou resultado pior do que a calha do mesmo transecto.
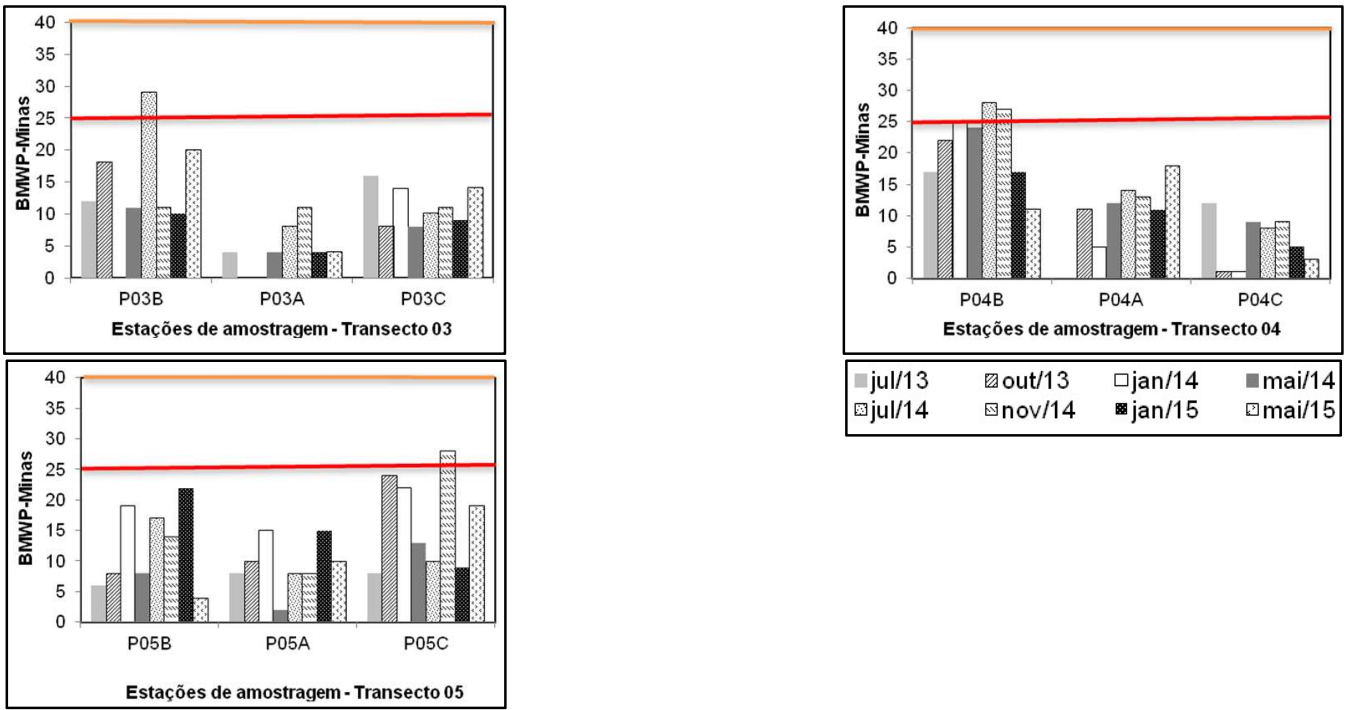

Figura 5: Variação espaço-temporal do BMWP Minas na rede amostral do reservatório de Volta Grande entre julho/13 e maio/15 
As correlações entre os índices de qualidade de água e bióticos são apresentadas na tabela 1. Dentre todas as correlações, apenas aquela entre a razão Calanoida/Cyclopoida e o BMWP/Minas foi significativa, ainda que o coeficiente seja baixo. Também é possível verificar que a correlação negativa (ainda que marginalmente significativa) entre o IET e o coeficiente múltiplo contradiz estes dois índices, pois em ambos, os baixos valores indicam oligotrofia.

Tabela 1: Matriz de correlações entre índices de qualidade de água e bióticos para o reservatório de Volta Grande. Coeficiente de correlação de Spearman encontra-se na diagonal da esquerda, enquanto o valor p encontra-se na diagonal direita. Valores em negrito indicam correlações significativas.

\begin{tabular}{|l|l|l|l|l|}
\hline Spearman/Valor p & IET & Coef. Múltiplo & Razão Cal/Cyc & BMWP/Minas \\
\hline IET & & 0,0657 & 0,9001 & 0,9671 \\
\hline Coef. Múltiplo & $-0,2181$ & & 0,1536 & 0,5962 \\
\hline Razão Cal/Cyc & 0,0193 & $-0,2163$ & & $\mathbf{0 , 0 1 9 3}$ \\
\hline BMWP/Minas & 0,0049 & 0,0635 & $\mathbf{- 0 , 3 4 7 6}$ & \\
\hline
\end{tabular}

\section{DISCUSSÃO}

Apesar dos parâmetros físico-químicos classificarem o reservatório de Volta Grande com boa qualidade, as comunidades biológicas retratam o ambiente como impactado. Dentre os principais impactos que as barragens causam na biota está alteração do regime hídrico, o que elimina os trechos de corredeiras, reduzindo a heterogeneidade ambiental e a diversidade das espécies, especialmente peixes e macroinvertebrados. A qualidade do habitat no reservatório de Volta Grande é ainda mais comprometida, devido à dragagem de areia em seu leito e aos sedimentos lodosos derivados da erosão no entorno.

Analisando os valores do IET, os pontos $4 \mathrm{~A}$ e $5 \mathrm{~A}$ apesar de serem considerados oligotróficos, são de especial atenção para a gestão do reservatório uma vez que no $4 \mathrm{~A}$ encontra-se um vasto banco de Egeria najas cuja decomposição pode causar liberação maciça de fósforo na coluna d'água. O ponto 5A está sob influência da planta da Vale Fertilizantes e do Ribeirão Conquistinha, cuja bacia drena parte do município de Delta/MG.

Quanto ao fitoplâncton, os organismos da classe Cryptophyceae são oportunistas, se desenvolvendo, principalmente, sobre condições adversas a outras espécies, como ambientes heterotróficos (Geraldes et al., 2005), fato esse que justifica a ocorrência dos mesmos. Além disso, é comum algas como diatomáceas e clorofíceas serem encontradas em maior quantidade nos trechos fluviais, como no transecto 5.

A comunidade zooplanctônica, refletiu influência das condições hidrodinâmicas típicas de reservatórios, com predomínio de Protozoa e náuplios no trecho lótico, onde observa-se maior vazão. Nos trechos intermediários (transectos 3 e 4), com a gradativa redução da velocidade da corrente, juvenis e adultos de Copepoda, as populações se mantém mais estáveis (Velho et al., 2005). A influência de afluentes como o Rio do Carmo e córrego Ponte Alta - cujas bacias sofrem influência de intensa atividade agrícola e urbana, respectivamente - se refletiu na razão Calanoida/Cyclopoida que classificou a maioria dos pontos como mesotróficos, principalmente no transecto 4.

Os macroinvertebrados foram mais abundantes nas margens quando comparado aos pontos amostrados na calha central. Nas margens a velocidade de correnteza é menor, bancos de macrófitas e mata ciliar (ainda que escassa) aumentam a heterogeneidade ambiental e a disponibilidade de recursos 
alimentares e substrato fornecendo maior quantidade de matéria orgânica (CORBI, 2006; SURIANO, 2008). No ponto 4A, a dominância de gastrópodes pode ser explicada devido à presença do extenso banco de $E$. cf. najas, com abundante perifíton associado, que fornece alimento a este grupo de organismos raspadores. Já no ponto $4 \mathrm{C}$, a grande quantidade de organismos da classe Oligochaeta, está relacionada às altas cargas de matéria orgânica e de sólidos.

A baixa frequência e abundância de organismos bentônicos fragmentadores refletem a ausência ou empobrecimento da vegetação ciliar, cujos detritos servem de recursos alimentares para este grupo (BUSS et al., 2002). Por outro lado, a grande abundância de filtradores e raspadores indica que há aumento progressivo no grau de trofia e grande quantidade de matéria orgânica particulada fina na água servindo de alimento para estes grupos (MANDAVILLE, 2002).

Em relação aos resultados do BMWP, a comunidade bentônica apresenta respostas a impactos ainda mais profundos do que o indicado por este índice, uma vez que o mexilhão-dourado (Limnoperma fortunei) é uma das espécies dominantes e não sendo contemplada pelo mesmo. Por classificar a comunidade sob os aspectos ecológicos, os grupos funcionais foram mais eficazes para explicar as alterações na comunidade bentônica quando comparado ao BMWP, que utiliza uma abordagem taxonômica.

A correlação entre índices de qualidade de água deve ser examinada com cautela, pois dependendo da faixa de variação de suas classificações, a pontuação baixa pode indicar boa qualidade de água (como IET, coeficiente múltiplo e razão Calanoida/Cyclopoida) ou má qualidade (no caso do BMWP). Tendo este fator em conta, é possível avaliar a relação entre estes índices.

Por ser negativa, a correlação entre a razão Calanoida/Cyclopoida e o BMWP/Minas indica que pontos considerados oligotróficos e propícios para copépodes da ordem Calanoida, podem apresentar baixa diversidade de táxons de macroinvertebrados bentônicos. De fato, reservatórios oligotróficos são caracterizados por espécies de fitoplâncton unicelulares e de alto valor nutricional para estes microcrustáceaos (BRITO et al., 2011) que ocorrem principalmente, na região limnética e/ou com baixa concentração de matéria orgânica. Estas condições são desfavoráveis para organismos bentônicos, especialmente os filtradores (VALDOVINOS et al., 2000).

A discrepância na correlação negativa entre IET e coeficiente múltiplo (marginalmente significativa) evidencia que apenas os parâmetros físicos e químicos não são suficientes para caracterizar um ecossistema. Ou seja, no reservatório de Volta Grande, os pontos classificados como oligotróficos pelo IET, onde se espera observar maior diversidade de espécies fitoplanctônicas, muitas vezes foram dominados por fitoflagelados (Criptophyceae). O coeficente múltiplo, ao incluir em seu cálculo diversas classes de fitoplâncton, considera esta diversidade relacionada à oligotrofia de ambientes aquáticos (REYNOLDS, 2002).

Uma limitação severa do uso da abordagem de bioindicadores é que não se tem para o reservatório de Volta Grande, nem para a grande maioria dos ambientes impactados por ação humana, pontos de referência com os quais compararem os resultados atuais. Tal ponto de referência deveria consistir num local onde não tivesse ocorrido nenhum tipo de alteração ambiental, nem sequer o próprio enchimento do reservatório há anos atrás. 
A fauna e flora desse local hipotético não estariam sujeitas ao processo de eutrofização recente, nem ao efeito da introdução de espécies exóticas, sendo consideradas prístinas do ecossistema (STODDARD, et al., 2006). Portanto, numa situação ideal, a comparação entre a biota dos locais de referência e a biota registrada nos pontos de amostragem de cada transecto daria a medida precisa e exata do quanto as comunidades que sofreram os efeitos dos impactos se diferenciaram das comunidades originais, ou seja perdendo espécies ou alterando a sua composição funcional.

A vegetação ripária escassa ou ausente reduz a entrada de matéria orgânica particulada e, somada à ausência de um ciclo sazonal natural no regime hídrico, afeta o fluxo de energia, reduzindo a diversidade dos grupos funcionais mais especializados e aumentando a abundância de onívoros (KARR, 2006). Finalmente, as interações bióticas são alteradas, não só na estrutura trófica do ecossistema, mas também pela perda de biodiversidade causada por competição com espécies exóticas.

Segundo Karr et al. (1986) existem cinco principais dimensões de qualidade ambiental dos ecossistemas aquáticos: qualidade da água, fluxo de energia, estrutura física do habitat, regime hídrico e interações bióticas. No caso do reservatório de Volta Grande, pode se concluir que quatro destas cinco dimensões foram drasticamente modificadas, quando da construção da barragem e da introdução de espécies exóticas ao sistema.

Os vários índices e indicadores utilizados fornecem respostas diferentes para a avaliação física, química e biológica, quando se considera apenas um dos compartimentos ou comunidades no ambiente aquático. Segundo Souza (2013), as diferenças observadas devem-se aos critérios e metodologias empregados na criação dos mesmos. De acordo com a autora, os índices podem até apontar diferenças expressivas entre ambientes com divergentes situações de conservação/degradação, mas muitas vezes não é sensível o suficiente para demonstrar diferenças na qualidade de água de um mesmo ambiente ao longo do tempo.

\section{CONCLUSÕES}

A comparação de indicadores da comunidade biológica no reservatório de Volta Grande, Rio Grande MG/SP permitiu concluir que a aplicação desses índices em conjunto traz um resultado com maior grau de assertividade, uma vez que a boa qualidade físico-química da água não reflete a degradação das comunidades biológicas. A avaliação simultânea dos dois principais compartimentos que determinam o funcionamento do ecossistema lótico (coluna d' água e sedimento de fundo) fornece um retrato mais detalhado e preciso, já que reflete alterações ambientais de curto prazo, registradas pelo fito e zooplâncton, e às quais os zoobentos não necessariamente são muito sensíveis e também alterações de longo prazo, registradas pelos zoobentos, e às quais o fitoplâncton, devido ao seu curto ciclo de vida, não responde.

\section{REFERÊNCIAS}

BRITO, S. L.; BARBOSA, P. M. M.; COELHO, R. M. P.. Zooplankton as an Indicator of Trophic Conditions in Two
Large Reservoirs in Brazil. Lakes and Reservoirs, v.16, p.253264, 2011. 
BUSS, D. F.; BAPTISTA, D. F.; SILVEIRA, M. P.. Influence of water chemistry and enviromental degradation on macroinvertebrate assemblages in a river basin in south-east Brazil. Hydrobiologia, v.481, n.3, 2002. DOI: http://doi.org.10.1023/A:1021281508709

CALLISTO, M.; GONÇALVES, G. F.; MORENO, P.. Invertebrados aquáticos como bioindicadores. In: GOULART, E. M. A.. Navegando o Rio das Velhas das Minas aos Gerais. Belo Horizonte: UFMG, 2005. p.555-556.

CALLISTO, M.; MORETTI, M.; GOULART, M..

Macroinvertebrados bentônicos como ferramenta para avaliar a saúde de riachos. Revista Brasileira de Recursos Hídricos, v.6, n.1, p.71-82, 2001.

CETEC. Centro Tecnológico. Padronização e consolidação metodológica de um índice biótico de qualidade de água para ambientes lóticos: Bacia do rio paraíba do sul. Belo Horizonte: FAPEMIG, 2007.

COELHO-BOTELHO, M. J.. Dinâmica da Comunidade Zooplanctônica e sua Relação com o Grau de Trofia em Reservatórios. São Paulo: CETESB, 2003.

COPAM. Conselho Estadual de Política Ambiental. Deliberação Normativa Conjunta COPAM/CERH-MG n.1 de 05 de maio de 2008. Dispõe sobre a classificação dos corpos de água e diretrizes ambientais para o seu enquadramento, bem como estabelece as condições e padrões de lançamento de efluentes, e dá outras providências. Belo Horizonte: COPAM, 2008

CORBI, J. J.. Influência de práticas de manejo de solo sobre os macroinvertebrados aquaticos de córregos: ênfase para o cultivo de cana-de-açúcar em áreas adjacentes. Tese (Doutorado) - Universidade Federal de São Carlos, São Carlos, 2006.

ELETROBRÁS FURNAS. Furnas Centrais Elétricas. Seminário Mexilhão Dourado: Atuação de Furnas no Rio Grande. Passos: MME, 2012.

ESTEVES, F. A.. Fundamentos de Limnologia. 3 ed. Rio de Janeiro: Interciência, 2011.

GANNON, J. E.; STEMBERGER, R. S.. Zooplankton (especially crustaceans and rotifers) as indicators of water quality. Transactions of the American Microscopical Society, v.97, n.1, p.16-35, 1978.

GERALDES, A. M.; BOAVIDA, M. J.. Seasonal water level fluctuations: Implications for reservoir limnology and management. Lakes \& Reservoirs, v.10, p.59-69, 2005.

GOULART, M.; CALLISTO, M.. Bioindicadores de qualidade de água como ferramenta em estudos de impacto ambiental. Revista da FAPAM, v.2, n.1, 2003.

HILSENHOFF, W.. Rapid field assesment of organic pollution with a family level biotic index. Journal of the North American Benthological Society, v.7, p.65- 68, 1988.

IGTEC. Instituto de Geoinformação e Tecnologia. Mapeamento do uso do solo e cobertura vegetal da bacia hidrográfica de contribuição lateral do reservatório de Volta Grande/MG. Belo Horizonte: IGTEC, 2015.
KARR, J. R.; FAUSCH, K. D.; ANGERMEIER, P. L.; YANT, P. R.; SCHLOSSER, I. J.. Assessment of biological integrity in running water: a method and its rationale. Springfield: Illinois Natural History Survey, 1986.

KELLY, M. G.; WHITTON, B. A.. The Trophic Diatom Index: a new index for monitoring eutrophication in rivers. Journal of Applied Phycology, v.7, p.433-444, 1995.

KLAVENESS, D.. Ecology of the Cryptomonadida: a first review. In: SANDGREEN, C. D.. Growth and reproductive strategies of freshwater phytoplankton. Cambridge: Cambridge University Press, 1988.

LAMPARELLI , M. C.. 2004. Grau de trofia em corpos d'água do estado de São Paulo: avaliação dos métodos de monitoramento. Tese (Doutorado em Ciências) Universidade de São Paulo, São Paulo, 2004.

MANDAVILLE, S. M.. Benthic macroinvertebrates in freshwaters: taxa tolerance values, metrics and protocols: (Project H-1). Soil \& Water Conservation Society of Metro Halifax, p.1-128, 2002.

MARQUES, M. G. S. M.; FERREIRA, R. L.; BARBOSA, F. A. R.. A comunidade de macroinvertebrados aquáticos e características limnológicas das Lagoas Carioca e da Barra, Parque Estadual do Rio Doce, MG. Revista Brasileira de Biologia, v.59, n.2, p.203-210, 1999.

METCALFE, J. L.. Biological water quality assessment of running waters based on macroinvertebrate communities: history and present status in Europe. Environmental Pollution, v.60, n.1, p.101-139, 1989.

NYGAARD, G.. Hidrologycal studies in some ponds and lakes part 2: The quotient hypothesis and some new or little known phytoplankton organisms. Biologiske Skrifte Det Kongelige Danske Videnskabernes Selskab Bind, v.7 n.1, p.1-293, 1949.

REECE, P. F.; RICHARDSON, J. S.. Biomonitoring with the reference condition approach for the detection of aquatic ecosystems at risk. In: DARLING, L. M.. Proceedings of a conference on the biology and management of species and habitats at risk. Kamloops: University Thompson Rivers, 1999. p.15-19.

REYNOLDS, C. S.. The development of perceptions of aquatic eutrophication and its control. Ecohydrology and Hydrobiology, v.3, n.2, p.149-163, 2002.

ROSENBERG, D. M.; RESH, V. H.. Introduction to freshwater biomonitoring and benthic macroinvertebrates. In: ROSENBERG, D. M.; RESH, V. H.. Freshwater biomonitoring and benthic macroinvertebrates, New York, 1993. p.1-9.

ROUND F. E.. Diatoms in river water: monitoring studies. Journal of Applied Phycology, v.3, p.129-145, 1991.

SLÁDECK, V.. Rotifers as indicators of water quality. Hydrobiologia, v.100, p.169-201, 1983.

SOUZA, A. P.. Avaliação da utilização de índices de integridade biótica do fitoplâncton como ferramenta para estimativa de qualidade da água nos Lagos Paranoá e Descoberto, no Distrito Federal. Dissertação (Mestrado) Universidade de Brasília, Brasília, 2013. 
STODDARD, L. J.; LARSEN, P. D.; HAWKINS, C. P.; JOHNSON K. R.; NORRIS, H. R.. Setting expectations for the ecological condition of Streams: the concept of reference condition. Ecological Applications, v.16, n.4, 2006, p.1267-1276, 2006.

SURIANO, M. T.. Macroinvertebrados em córregos de baixa ordem sob diferentes usos do solo no estado de São Paulo: subsídios para o biomonitoramento. São Carlos: UFSCar, 2008.

TUNDISI, J. G.; MATSUMURA-TUNDISI, T.; HENRY, R.; ROCHA, O.; HINO, K.. Comparação do estado trófico de 23 reservatórios do Estado de São Paulo: eutrofização e manejo. In: TUNDISI, J. G.. Limnologia e manejo de represas. São Paulo: 1988.
VALDOVINOS, C.; FIGUEROA, R.. Benthic community metabolism and trophic conditions of the South American lakes. Hydrobiologia, v.429, p.151-156, 2000.

VELHO, L. F. M.; LANSAC-TOHA, F. A.; BONECKER, C. C..

Distribuição Longitudinal da Comunidade Zooplanctônica em Reservatórios. In: RODRIGUES, L. S. M.; THOMAZ, A. A..

AGOSTINHO, L. C. G.. Biocenoses em Reservatórios: padrões espaciais e temporais. São Carlos: Editora Rima, 2005. p.129145.

WARD, D.; HOLMES, N.; JOSÉ, P.. The New Rivers \& Wildlife Handbook. Bedfordshire: RSPP, NRA and The Wildlife Trusts, 1995. 